\title{
Interpretando processos comportamentais básicos em um paradigma neuropsicológico de processamento de expressões faciais de emoção
}

\author{
Interpretación de los procesos básicos de comportamiento en un \\ paradigma neuropsicológico de procesamiento de expresiones faciales \\ de la emoción
}
Interpreting basic behavioral processes in an emotional face processing neuropsychological paradigm

Pedro Fonseca Zuccoloํ, Felipe D'Alessandro Corchs ${ }^{2}$ e Denigés Regis Maurel Neto

\begin{abstract}
[1] Laboratório de Análise Biocomportamental, Departamento de Psicologia Experimental, Instituto de Psicologia da USP; Núcleo Paradigma de Análise do Comportamento [2] Núcleo Paradigma de Análise do Comportamento; Departamento e Instituto de Psiquiatria do HC FM USP [3] PUC-SP I Título abreviado: Processos comportamentais no reconhecimento de faces I Endereço para correspondência: Rua Prof. Mello Moraes, 1721 Bloco A Sala C6 - Butantã CEP: 05508-030 - São Paulo, SP - Brasil I Email: pedrozuccolo@yahoo.com.br
\end{abstract}

Resumo: A interpretação de pesquisas em neurociências sob uma ótica analítico-comportamental pode ser útil para analistas do comportamento, permitindo a importação de tecnologias desenvolvidas nessas áreas para o estudo do comportamento sob enfoque analítico-comportamental. Além disto, as explicações de outras tradições teóricas são baseadas em eventos comportamentais, trazendo dados de interesse ao analista do comportamento. Este trabalho teve por objetivo analisar um tema explorado pelas neurociências, qual seja, o chamado processamento de expressões faciais de emoção. Um dos muitos procedimentos usados no estudo do processamento de expressões faciais de emoção foi analisado a partir das seguintes perguntas: quais seriam as relações funcionais entre estímulos e respostas avaliadas por esse instrumento? Como ele poderia ser utilizado em pesquisas com abordagem analítico-comportamental? Analisamos a Tarefa de Reconhecimento de Expressões Faciais (Harmer, Rogers, Tunbridge, Cowen \& Goodwin, 2003), teste computadorizado no qual os sujeitos têm de classificar o mais rápida e precisamente possível faces humanas expressando diferentes emoções. A análise foi feita a partir de conceitos que se referem a formas de classificação do comportamento e processos comportamentais básicos. Argumentamos que essa tarefa poderia ser entendida como um teste que avalia controle de estímulos envolvidos em interações sociais, especificamente, como um análogo ao teste de generalização, no qual o sujeito nomeia estímulos para os quais foi treinado a responder diferencialmente ao longo da vida. A partir dessas interpretações, são levantados problemas de pesquisa que poderiam ser respondidos utilizando esse procedimento.

Palavras-chave: Análise do Comportamento, testes neuropsicológicos, expressões faciais, controle de estímulos

Abstract: Interpreting research in the field of neuroscience from a behavioral-analytic framework may be useful for behavior analysts, as it may help adapting technology developed outside the discipline for studying phenomena from the behavior analysis perspective. 
Furthermore, explanations for behavior originated in other theoretical traditions are based on behavioral phenomena and as such bring data of interest for the behavior analyst. Our objective was to analyze a widely explored subject in neuroscience, namely, the processing of emotional faces. One of the many experimental procedures used in the study of emotional face processing was analyzed based on the following questions: what are the functional relations between stimuli and responses that are assessed by this instrument? How could it be used in research with a behavioral-analytic perspective? We analyzed the Facial Recognition Task (Harmer, Rogers, Tunbridge, Cowen \& Goodwin, 2003), a computerized test in which subjects must categorize human faces expressing different emotions as fast and as accurately as possible. The analysis of the task was conducted using concepts from behavior analysis related to classification of behavior and basic behavioral processes. We argue that this task may be seen as a test assessing stimulus control of information present in social interactions, specifically, as an analogue to the generalization gradient in which subjects must name stimuli they have been trained to respond to differentially throughout life. Several behavioral-analytic research questions for which this task may be useful are described in the conclusion.

Key words: behavior analysis, neuropsychological tests, facial expression, stimulus control.

Resumen: La interpretación de investigaciones en neurociencias bajo una óptica analíticoconductual puede resultar útil para analistas del comportamiento al permitir la importación de tecnologías desarrolladas en esas áreas para el estudio del comportamiento bajo un enfoque analítico-conductual. Por otra parte, las explicaciones de otras tradiciones teóricas están basadas en eventos conductuales que aportan datos de interés para el analista del comportamiento. Este trabajo tuvo como objetivo analizar un tema explorado por las neurociencias, el llamado procesamiento de expresiones faciales de la emoción. Se analizó uno de los muchos procedimientos usados en el estudio del procesamiento de expresiones faciales de la emoción a partir de las siguientes preguntas: ¿cuáles serían las relaciones funcionales entre estímulos y respuestas evaluadas por este instrumento? ¿Cómo podría ser utilizado en investigaciones con abordajes analítico-conductuales? Analizamos la Tarea de Reconocimiento de Expresiones Faciales (Harmer, Rogers, Tunbridge, Cowen \& Goodwin, 2003), test computadorizado en el cual los sujetos tienen que clasificar, lo más rápida y precisamente posible rostros humanos que expresan diferentes emociones. El análisis se hizo a partir de conceptos que se refieren a formas de clasificación de la conducta y a procesos de comportamiento básicos. Argumentamos que se podría entender esa tarea como un test que evalúa el control de estímulos implicados en interacciones sociales, específicamente, como análogo al test de generalización, en el cual el sujeto nombra estímulos para los que fue entrenado a responder de forma diferente a lo largo de la vida. A partir de estas interpretaciones, se hace un relevamiento de problemas de investigación que se podrían responder utilizando ese procedimiento.

Palabras clave: Análisis del comportamiento, testes neuropsicológicos, expresiones faciales, control de estímulos. 
A interpretação de pesquisas e teorias de outras tradições da Psicologia de um ponto de vista analítico-comportamental tem sido apontada como uma atividade útil para o analista do comportamento (Corchs, 2010; de Rose, 1999; Ferster, Culbertson \& Boren, 1968/1977; Green, 2006; Lattal, 2005, 2008; Marr, 1984; Strapasson, Carrara \& Lopes Júnior, 2007). No nível teórico, a leitura de outras tradições da psicologia gera um desafio ao analista do comportamento, que, ao lidar com problemas apontados por outras disciplinas, pode ter sua posição fortalecida ou, caso se mostre necessário, abandonada (Marr, 1984). A interpretação comportamental de termos ou conceitos de outras tradições da psicologia pode ainda ter uma função heurística na investigação de relações comportamentais possivelmente negligenciadas por analistas do comportamento (Strapasson et al., 2007). Além disso, embora outras concepções teóricas expliquem o comportamento de maneiras diferentes, estas explicações são formuladas a partir de eventos comportamentais e, portanto, podem trazer dados de interesse ao analista do comportamento (Catania, 1999; de Rose, 1999). No nível aplicado, a leitura de outras tradições científicas (incluindo outras tradições da psicologia) pode permitir a importação de procedimentos potencialmente úteis para o estudo do comportamento sob uma perspectiva analítico-comportamental (Green, 2006; Lattal, 2005, 2008).

Esse é particularmente o caso da produção na psicologia cognitiva e também da neuropsicologia, que produzem dados a partir da experimentação e manipulação de variáveis, atividades estas tão importantes para um analista do comportamento (Catania, 1999; Lezak, Howieson \& Loring, 2004). Além disso, trabalhos nessas áreas comumente pesquisam temas para os quais a Análise do Comportamento não tem uma resposta definitiva (Wray, Freund \& Dougher, 2009). Um desses temas é o do reconhecimento de expressões faciais de emoções.

O estudo da associação entre emoção e expressões faciais remonta aos trabalhos de Darwin (1872) sobre expressões faciais em homens e animais. A maior parte dos dados produzidos sobre o tema, contudo, concentra-se nos últimos trinta anos, sob influência sobretudo das pesquisas do psicólogo americano Paul Ekman (1934- ). Ekman e colaboradores conduziram uma séries de investigações em diferentes países e culturas do mundo a respeito de como as pessoas classificam determinadas expressões faciais de emoções (Ekman \& Friesen, 1971; Ekman, Friesen \& Tomkins, 1971). Em suas pesquisas, Ekman e colegas observaram que expressões faciais de algumas emoções ditas "básicas" são classificadas de maneira muito similar entre culturas, mesmo em comunidades onde não há linguagem escrita (Andrew, 1963; Ekman, 1972, 1973; Ekman \& Friesen, 1971; Ekman, Sorenson \& Friesen, 1969). Esses dados levaram à hipótese de que há uma universalidade nas expressões faciais de emoção, no sentido de uma conexão universal entre configurações faciais particulares e emoções específicas (Ekman, 1992, 1999; Ekman et al., 1987). Alegria, tristeza, raiva e nojo são consideradas emoções básicas, cada qual tendo uma expressão facial específica (Ekman, 1999); embora haja algumas controvérsias, surpresa também é considerada uma emoção básica (Ekman, 1999).

A questão da conexão entre emoções e expressões faciais é complexa e cheia de nuances. Embora os dados de Ekman e colaboradores sugiram um aspecto universal nas expressões faciais de emoção, há autores cuja posição é contrária à essa hipótese (e.g., Russel, 1994). Além disso, tanto a produção de uma expressão facial como o seu reconhecimento por um terceiro podem ser afetados por diversos fatores. A hipótese de Ekman $(1979,1999)$ é que a conexão universal entre expressões e emoções básicas foi estabelecida por meio da seleção natural, porém o próprio autor observou que a cultura (e, em especial, a linguagem), o agrupamento social dentro de culturas e algumas diferenças individuais relacionados à história de vida podem produzir diferenças na expressão de expressões faciais de emoções, no que uma determinada expressão significa para as pessoas que a mostram e o seu reconhecimento por terceiros (Ekman, 1992, 1999). Uma discussão detalhada dessas questões exigiria um trabalho à parte e está além do escopo desse artigo. O leitor interessado pode encontrar mais detalhes em Ekman (1992, 1999), Ekman e Friesen (1971) e Russel (1994).

De interesse para este trabalho é que os estudos de Ekman e colegas levaram ao desenvolvimento de um banco de dados de fotografias de atores expres- 
sando essas emoções básicas (i.e., Picture of Facial Affect, de Ekman \& Friesen, 1976), amplamente usado em paradigmas do chamado processamento emocional, condicionamento aversivo e em investigações usando neuroimagem funcional (Del-Ben, 2008; Fusar-Poli et al., 2009)'.

Atualmente, há um grande número de pesquisas na área das neurociências que utilizaram o banco de dados de fotografias de Ekman e Friesen para entender os sistemas cerebrais envolvidos no reconhecimento de expressões faciais das emoções ditas básicas. Estudos nessa área têm mostrado que o reconhecimento de emoções em expressões faciais pode ser afetado por alterações em sistemas cerebrais específicos como, por exemplo, o sistema serotoninérgico (Attenburrow et al., 2003; Browning, Reid, Cowen, Goodwin \& Harmer, 2007; Harmer, 2008; Harmer, Bhagwagar, et al., 2003; Harmer, Rogers, Tunbridge, Cowen e Goodwin, 2003) ou noradrenégico (Harmer, Hill, Taylor, Cowen \& Goodwin, 2003; Harmer, Shelley, Cowen, \& Goodwin, 2004). Outras pesquisas mostram que o reconhecimento de faces pode variar em função do sexo ou de acordo com a fase do ciclo menstrual (Guapo et al., 2009). É notável também a existência de pesquisas com participantes com transtornos psiquiátricos (por exemplo, Depressão - Hayward, Goodwin, Cowen \& Harmer, 2005; Surguladze et al., 2004), mostrando que sujeitos com determinados diagnósticos psiquiátricos apresentam algumas diferenças em relação à sujeitos não diagnosticados no reconhecimento de emoções em faces.

Esses estudos mostram alterações no reconhecimento de diferentes expressões faciais de emoção quando das manipulações nos sistemas cerebrais citadas acima ou em função de sexo ou doenças psiquiátricas. Contudo, a única expressão facial de emoção cujo reconhecimento é afetado em todas essas situações de modo consistente é o medo (DelBen, Alves-Neto \& Graeff, 2008). As explicações para esse achado e os usos dele para formulações

1 Um aspecto importante a salientar é que o estudo da associação entre expressões faciais e emoção tem sido feito de diversas perspectivas (psicológicas, neuropsicológicas, antropológicas etc). Citamos apenas os trabalhos na área da neuropsicologia e neurociências porque são essas as áreas que pesquisam a relação entre faces, emoções e problemas clínicos, o que é o foco de interesse dos autores. teóricas fogem dos objetivos desse artigo, porém é importante citar que esses dados têm sido usados para explicar a gênese e manutenção de alguns transtornos psiquiátricos tais como ansiedade e depressão e os mecanismos pelos quais os tratamentos farmacológicos para eles funcionariam ${ }^{2}$ (para mais detalhes sobre essa discussão, sugere-se a leitura de Harmer, 2008).

Da mesma maneira que as pesquisas em neurociências buscam entender os mecanismos neurais subjacentes ao reconhecimento de faces de emoções, poderíamos nos perguntar a respeito da influência de variáveis ambientais sobre esse comportamento. Por exemplo, o reconhecimento de expressões faciais pode variar em função da exposição a diferentes contingências de reforçamento e/ ou controle aversivo? Há diferenças no reconhecimento de expressões faciais de emoção de acordo com diferentes histórias de vida?

Os paradigmas neuropsicológicos usados nas pesquisas acima são baseados em comportamentos observados e construídos a partir de dados experimentais e empíricos. Ao entender os processos comportamentais envolvidos nesses paradigmas, analistas do comportamento podem interpretar pesquisas em neurociências sob uma perspectiva analítico-comportamental. Além disto, o entendimento dos processos comportamentais envolvidos nesses paradigmas poderia abrir a possibilidade de seu uso em pesquisas com enfoque teórico analítico-comportamental.

Considerando a grande variedade de procedimentos utilizados nas pesquisas em neurociências e o escopo deste trabalho, optamos por selecionar

2 Essas formulações foram foram feitas com base na hipótese de que faces de medo seriam um estímulo "aversivo" (também referido como um estímulo com "valência emocional negativa”) (Darwin, 1872; Ekman, Sorenson \& Friesen, 1969). Porém, o termo "aversivo", da maneira como é usado na literatura em neurociências, pode ter um significado diferente daquele usada na Análise do Comportamento, pois se refere à hipótese de que faces de medo podem sinalizar perigos oriundos do ambiente. Contudo, ainda não está estabelecido se esse estímulo teria valor de reforçador negativo, um dos critérios utilizados em Análise do Comportamento para considerar um estímulo como aversivo (Hunziker, 2011) Sabemos que esse é um assunto que merece atenção, porém ele não será abordado aqui por não fazer parte dos nossos objetivos para este trabalho. 
a Tarefa de Reconhecimento de Expressões Faciais (Harmer, Bhagwagar, et al., 2003; Harmer, Hill, et al., 2003; Harmer, Rogers, et al., 2003; Harmer et al., 2004). A escolha desse procedimento específico ocorreu por algumas razões: ele tem sido utilizado também como um instrumento objetivo que não depende de autorelato na medida de variáveis emocionais centrais em processos depressivos e ansiosos (Harmer, 2008). Ademais, essa medida se mostrou confiável e com elevado valor preditivo de resposta terapêutica. Por exemplo, estudos utilizando a Tarefa de Reconhecimento de Expressões Faciais detectaram diferenças entre os sujeitos examinados que predisseram quem ia melhorar com tratamento com antidepressivos quinze dias depois (Harmer, 2008). Essas alterações no desempenho foram detectadas mesmo quando a pessoa não era capaz de descrever mudanças em suas reações emocionais por meio de relato ou escalas (Harmer, 2008).

O objetivo deste trabalho foi responder às seguintes perguntas: quais seriam as possíveis relações funcionais entre estímulos e respostas envolvidas nesse instrumento? De que maneira esse instrumento poderia ser utilizado em pesquisas que se utilizem de um enfoque analítico-comportamental?

\section{Método}

O paradigma neuropsicológico denominado Tarefa de Reconhecimento de Expressões Faciais (Facial Expression Recognition Task) foi analisado com base nas informações contidas nas publicações que se utilizaram dele (Harmer, Bhagwagar, et al., 2003; Harmer, Hill, et al., 2003; Harmer, Rogers, et al., 2003; Harmer et al., 2004; Young et al., 1997).

Como categorias de análise, foram escolhidos conceitos que dizem respeito a formas de classificação do comportamento quando do seu estudo sob uma ótica analítico-comportamental e conceitos relacionados a processos comportamentais básicos. A escolha das categorias se deu da seguinte forma: primeiro foram eleitos conceitos relacionados à classificação do comportamento, a partir dos quais foi realizada uma primeira análise dos dados. Essa primeira análise gerou hipóteses a respeito de processos comportamentais básicos possivelmente envolvidos no procedimento examinado. Os concei- tos referentes a esses processos básicos foram então apresentados e novamente se fez uma análise dos dados à luz destes.

Em vista da possibilidade de controvérsias quanto à definição de alguns conceitos básicos em análise do comportamento, optou-se por buscar as definições das categorias em um livro didático da área, mais especificamente Aprendizagem: Comportamento, Linguagem e Cognição, de Catania (1999). Esse livro foi escolhido por algumas razões: (a) é amplamente utilizado em cursos de graduação e pós-graduação, (b) abarca diversos temas, (c) é conceitualmente rigoroso, (d) inclui todas as categorias de análise escolhidas e (e) tem um glossário com a definição de vários termos e índice remissivo, facilitando a busca pelas categorias de análise e a descrição de seus pormenores.

Ao final desse processo, as seguintes categorias foram utilizadas na análise: (a) operações experimentais envolvidas no estudo do comportamento; (b) conceito de controle de estímulos do comportamento operante; (c) conceito de classe de ordem superior; e (d) nomeação.

A análise dos textos selecionados foi feita seguindo os seguintes passos:

(1) Descrição do procedimento metodológico a ser analisado a partir dos textos selecionados; (2) Classificação dos comportamentos envolvidos no teste neuropsicológico a partir da primeira categoria escolhida (operações experimentais). Essa análise envolveu as seguintes etapas: (a) leitura da categoria; (b) articulação desta com a descrição do teste neuropsicológico analisado; (c) levantamento de hipóteses a respeito de processos comportamentais envolvidos na tarefa;

(3) Leitura das novas categorias levantadas na etapa anterior como possíveis conceitos de análise. A partir disso, tentou-se novamente articular a descrição do teste neuropsicológico com os conceitos escolhidos;

(4) Proposição de perguntas de pesquisa a partir das hipóteses levantadas em 2 e 3 e questionamento da aplicabilidade da tarefa neuropsicológica analisada em estudos que tenham enfoque analítico-comportamental. 
Paradigma neuropsicológico analisado: Tarefa de Reconhecimento de Expressões Faciais (Facial Expression Recognition Task) A descrição que se segue é baseada nos trabalhos de Harmer e colaboradores (Harmer, Bhagwagar, et al., 2003; Harmer, Hill, et al., 2003; Harmer, Rogers, et al., 2003; Harmer et al., 2004). Para facilitar a compreensão do leitor, ela será dividida em subitens: estímulos, procedimento, variáveis analisadas e questões suscitadas pela descrição dos autores.

\section{Estímulos}

Os estímulos usados pelos autores foram fotografias de faces anônimas desenvolvidas por Ekman e Friesen (Picture of Affect Series - Ekman \& Friesen, 1976). Essas faces expressavam uma de seis emoções básicas: alegria, surpresa, tristeza, medo, raiva e nojo (em Inglês, respectivamente, happiness, surprise, sadness, fear, anger e disgust). Esses estímulos foram submetidos a um processo de transformação que consistiu no desenvolvimento de gradações para cada estímulo, de maneira que cada face teria diversas versões, desde uma expressão neutra ( $0 \%$ ) até o protótipo de uma determinada emoção básica (100\%).

Foram utilizadas fotografias dos rostos de 10 indivíduos diferentes. Os autores selecionaram quatro intensidades de cada emoção (gradações de $20 \%)$ para a fotografia de cada um dos rostos. Além disto, eles também selecionaram a fotografia com expressão neutra de cada rosto. Assim, o total de estímulos apresentados na tarefa foi de 250 .

\section{Procedimento}

Os estímulos eram apresentados aleatoriamente aos sujeitos em uma tela de computador por 500 milissegundos e substituídos por uma tela branca. Os sujeitos tinham de responder pressionando teclas rotuladas no teclado, quando a tela branca aparecia. Cada voluntário era solicitado a responder o mais rápida e precisamente possível, pressionando a tecla com o rótulo que correspondia à emoção que ele achava que estava sendo denotada pelo rosto. Não havia qualquer sinalização para o sujeito dizendo se ele estava respondendo de maneira correta ou não.

\section{Variáveis avaliadas}

As seguintes variáveis foram analisadas na tarefa: (a) Precisão: número de respostas corretas para cada emoção (de um total de 40). Se cada fotografia tinha quatro exemplares de cada emoção e eram 10 fotografias no total, então havia 40 estímulos que poderiam ser classificados em uma mesma categoria (e.g., alegria);

(b) Tempo de reação para escolhas corretas;

(c) Classificações erradas (i.e., número de respostas para cada categoria de expressão facial que era classificada pelo sujeito como pertencendo a qualquer uma das outras categorias de expressão facial).

\section{Instruções}

Não há nos trabalhos de Harmer e colaboradores (Harmer, Bhagwagar, et al., 2003; Harmer, Hill, et al., 2003; Harmer, Rogers, et al., 2003; Harmer et al., 2004) uma descrição detalhada das instruções dadas ao sujeitos, apenas descreve-se que os sujeitos são solicitados a classificar faces o mais rapidamente possível pressionando a tecla no teclado que corresponde à emoção que eles "acham" que está sendo mostrada.

\section{Resultados}

\section{Processos comportamentais possivelmente envolvidos na Tarefa de Reconhecimento de Expressões Faciais}

\section{Operações experimentais envolvidas na Tarefa de Reconhecimento de Expressões Faciais} Catania (1999) propõe que os vários fenômenos do comportamento ${ }^{3}$ podem ser descritos por meio

3 Em análise do comportamento, convencionou-se utilizar o termo comportamento para denominar uma relação entre aquilo que o organismo faz e os eventos ambientais que estavam presentes ou que se seguiram a essas ações (Skinner, 1981/2007). Já o uso do termo resposta foi restrito àquilo que o organismo faz (Skinner, 1953). Contudo, pelo fato de as principais categorias de análise escolhidas neste trabalho terem sido retiradas da obra de Catania (1999), optou-se por usar o termo comportamento do modo como este autor o utiliza. Assim, a partir deste ponto do trabalho, comportamento aqui poderá ser entendido em um sentido mais coloquial, 
de uma taxonomia comportamental que permite organizar os fatos do comportamento sobre os quais já se tem conhecimento e os diversos procedimentos que um experimentador realiza quando o estuda. Tudo aquilo que um experimentador faz durante o estudo do comportamento pode ser chamado de operação experimental; as mudanças no comportamento que ocorrem após essas operações podem ser denominadas processos comportamentais. Catania (1999) descreve cinco operações básicas que um experimentador pode realizar: (1) observação de comportamento, (2) apresentação de estímulos, (3) apresentação de estímulos como consequência, (4) sinalização de apresentações de estímulos ou sinalização da disponibilidade/possibilidade de certas consequências seguirem certas respostas e (5) estabelecimento da efetividade de consequências (p. 33).

De acordo com esse lógica, fala-se em (1) observação de comportamento de interesse quando examinamos este comportamento na sua relação com um dado contexto. Já a (2) apresentação de estímulos refere-se a observação da relação entre o aparecimento de certos estímulos e as respostas que o seguem. Envolve, por exemplo, observar quais são as características essenciais de certos estímulos que estão relacionados ao aparecimento de comportamentos de interesse. Assim, apresentar estímulos envolve mudar propriedades do ambiente e ver qual é a diferença na frequência (ou noutra propriedade do comportamento) que em geral ocorre após essa mudança (Catania, 1999).

O experimentador pode também (3) apresentar estímulos como consequências de uma dada resposta do organismo. Consequências podem variar desde a apresentação de eventos com significância biológica óbvia (e.g., apresentação de comida ou água) até mudanças menores em coisas vistas, ouvidas e tocadas. Além disso, consequências podem envolver alteração de estímulos já presentes, remoção ou prevenção de estímulos ou, ainda, mudanças nas consequências de outras respostas. Não basta observar uma única instância, mas sim um fluxo, de modo a avaliar os efeitos dessas consequenciações sobre este fluxo.

como "qualquer coisa que um organismo faça”, e poderá ser intercambiável com o termo resposta (Catania, 1999, p. 390).
Uma quarta operação ocorre quando estímulos se tornam sinalizadores efetivos, ou seja, quando eles (4) sinalizam a apresentação de outros estímulos ou a disponibilidade/possibilidade de certas consequências seguirem certas respostas. Operações que envolvem sinais são chamadas de operações de sinalização ou de controle de estímulos. A (2) apresentação de estímulos ou (3) consequenciação não ocorre isoladamente. Em geral, elas são sinalizadas por outros eventos. Assim, essas operações de sinalização podem ser combinadas com apresentação de estímulo ou operações de consequenciação. Quando combinadas a uma dessas operações, a sinalização terá uma função diferente: no primeiro caso, ela será (a) sinalização de apresentação de estímulos (por exemplo, quando um estímulo apresentado sinaliza o aparecimento de outro estímulo, este último eliciador de uma resposta reflexa); no segundo caso, ela será uma (b) sinalização de consequências, ou seja, ao invés de sinalizar a apresentação de um estímulo, um estímulo pode sinalizar quando uma resposta vai ter uma certa consequência caso seja emitida pelo organismo.

Por fim, Catania (1999) descreve que um experimentador pode (5) estabelecer a efetividade de consequências. As operações que podem ser feitas para mudar a efetividade de uma consequência são chamadas operações estabelecedoras. A diferença entre operações estabelecedoras e apresentação de estímulos discriminativos é a seguinte: estímulo discriminativo fornece a ocasião na qual certa resposta será seguida de certo reforço; operação estabelecedora faz com que certo estímulo torne-se importante como reforçador para certas respostas.

Quais operações estão presentes na Tarefa de Reconhecimento de Expressões Faciais? Algumas possibilidades foram levantadas a partir de uma análise descritiva da tarefa e do exame da qualidade dos estímulos nela envolvidos.

De um ponto de vista descritivo, a Tarefa de Reconhecimento de Expressões Faciais é uma circunstância na qual um sujeito é instruído verbalmente a classificar/nomear faces que lhe serão apresentadas e na qual estas são mostradas sucessivamente por um breve intervalo de tempo (500ms), sendo que o sujeito deve emitir uma resposta diante de cada estímulo; caso o sujeito não emita ne- 
nhuma resposta, a próxima fotografia não lhe é apresentada. $\mathrm{O}$ sujeito deve classificar as faces de acordo com a emoção que ele "pensa/acredita" estar sendo retratada em cada uma delas, sendo que não há consequência programada para as respostas do sujeito, a não a apresentação da nova face a ser reconhecida (ao sujeito não é explicitado se houve um acerto ou erro no reconhecimento da face). Partindo dessa descrição, poderíamos dizer que o experimentador realiza a observação do comportamento a partir da apresentação de estímulos, sem operações consequenciais programadas. Contudo, essa análise por si só é incompleta, sendo importante também examinar a qualidade dos estímulos apresentados. $\mathrm{O}$ fato dos estímulos serem faces exigem algumas considerações para a presente análise.

As expressões faciais fazem parte da vida humana e são condições da vida social. Portanto, são estímulos com os quais já devem existir diversos tipos de história prévia. O reconhecimento de expressões faciais de emoções tem sido apontado na literatura como importante para a adaptação e funcionamento social (Del-Ben, Ferreira, AlvesNeto \& Graeff, 2008). Atualmente, pensa-se que uma das funções da identificação de expressões faciais de emoções é o direcionamento das respostas de sujeitos envolvidos em uma interação social (Horstmann, 2003). Segundo Horstmann (2003), isto ocorre porque, do ponto de vista de quem observa, as expressões faciais fornecem informações a respeito de estados emocionais, intenções e/ou desejos de outrem. Além disso, as expressões faciais de emoção dão informações sobre as contingências que estão fazendo a pessoa responder com uma determinada mudança na face. De fato, a exposição às mais diversas expressões faciais ocorre desde as primeiras interações e ao longo de toda a vida, interações estas nas quais determinadas respostas podem produzir certos tipos de consequências a depender de contextos específicos.

Do ponto de vista daquele que observa uma expressão facial, esta pode ser considerada uma condição na qual uma resposta será seguida de certas consequências. Por exemplo, a expressão emocional de alegria por parte de alguém com quem se tem uma relação de intimidade pode ser uma condição na qual manifestações de afeto foram seguidas de demonstrações de reciprocidade.
O fato de expressões faciais de emoção fazerem parte das ocasiões nas quais algumas respostas são seguidas de certos tipos de consequências sugere que esses estímulos participam, em maior ou menor medida, de relações comportamentais operantes. Assim, voltando à questão das operações experimentais envolvidas na Tarefa de Reconhecimento de Expressões Faciais, é plausível considerar que mostrar diferentes faces é uma operação de apresentação de estímulos que sinalizam consequências que, embora ausentes nesta ocasião específica, estiveram presentes ao longo da vida. Segundo Catania (1999), quando falamos de operações de sinalização, falamos de controle de estímulos.

\section{Tarefa de Reconhecimento de Expressões Faciais: um teste de controle de estímulos?}

Frequentemente as respostas de um organismo produzem consequências somente quando ocorrem na presença de determinados estímulos (Catania, 1999). Nesse caso, quando o responder é reforçado apenas na presença desses estímulos, diz-se que o reforço é correlacionado ao estímulo (Catania, 1999). Para Catania (1999), uma classe de respostas criada por este reforçamento diferencial em relação às propriedades do estímulo é denominada operante discriminado. Nas palavras do autor:

Os operantes discriminados são um traço difuso do comportamento. Ao dirigir um carro, avançamos por um cruzamento se o semáforo estiver verde, mas não se ele estiver vermelho. Ao falar com alguém, o que dizemos é afetado pelo que a outra pessoa diz, por sua postura e expressão facial [ênfase adicionada], pela situação em que ocorre a conversa, e assim por diante. (pp. 145-146)

Os estímulos discriminativos correspondem àquilo que coloquialmente chamamos de sinais ou pistas e as ações comportamentais como atenção e percepção. Eles estabelecem a ocasião em que as repostas têm consequências, por isso diz-se que eles ocasionam as respostas (Catania, 1999).

Uma questão importante é que os estímulos correlacionados com o reforço podem ter dimensões ou propriedades variadas, de modo que um organismo pode ficar sob controle de uma deter- 
minada dimensão e não de outra propriedade ou dimensão do mesmo estímulo (Catania, 1999). Segundo Catania (1999), aqui o conceito de atenção é essencial porque organismos podem responder a algumas propriedades de estímulos, mas não a outras, sendo que as propriedades de estímulos às quais um organismo responde discriminativamente são chamadas de salientes. Considerar atenção como comportamento é importante, pois o atentar (para) pode ter consequências e, nos casos em que essas consequências alteram a frequência do atentar, podemos falar desse comportamento como um operante (Catania, 1999). De fato, existe toda uma área dentro da análise do comportamento em que se estuda a resposta de observação (Dinsmoor, 1983; Tomanari, 2009).

A questão da variabilidade das dimensões de um estímulo é importante porque as expressões faciais de emoções são estímulos (ou conjuntos de estímulos) extremamente complexos e variados. As propriedades do estímulo face podem ocorrer em diversas gradações, de modo a denotar desde uma expressão neutra até a expressão de uma emoção qualquer; ou então variar de uma emoção para outra, com diferentes combinações (Ekman, 1999). No caso da Tarefa de Reconhecimento de Expressões Faciais, não somente são apresentadas diversas expressões de emoções básicas diferentes, como também são mostradas diferentes gradações de uma mesma emoção.

De acordo com Catania (1999), a pesquisa sobre gradientes de controle de estímulo busca estudar justamente os efeitos de variações nas dimensões de um estímulo sobre uma determinada resposta. Os procedimentos envolvidos nesse tipo de pesquisa em geral envolvem as seguintes etapas: (a) treino, durante o qual é programada alguma correlação entre os estímulos e o reforço; (b) fase de teste, durante a qual o reforço é suspenso, enquanto os estímulos novos e velhos (ou variadas gradações do estímulo) são apresentados e o experimentador observa as respostas do sujeito aos diferentes estímulos (Catania, 1999). Os chamados gradientes ${ }^{4}$ de controle de estímulo são construídos nessa segunda fase.

4 Catania (1999) define gradiente como uma "medida do responder durante diferentes estímulos como uma função de sua localização ao longo de um contínuo. Os gradientes
A fase de teste de uma pesquisa sobre gradientes de controle de estímulo é importante justamente porque revela sob qual (ou quais) dimensão(ões) ou aspecto(s) de um estímulo uma classe de respostas passa a ser controlada (Catania, 1999). Além disto, esse teste também permite observar se o responder ocorre na presença de estímulos para os quais não houve treino, processo este denominado generalização (Catania, 1999).

É possível observar semelhanças entre a fase de teste de um estudo sobre gradientes de controle de estímulos e a Tarefa de Reconhecimento de Expressões Faciais. Isto porque esta é uma situação na qual se observa as respostas de um sujeito frente à apresentação de faces com diferentes emoções e em diversas gradações (variações nas propriedades do estímulo), sendo que o treino discriminativo ocorreu ao longo de toda a vida e por meio da interação com outros sujeitos. Assim, poderíamos levantar a possibilidade de o Teste de Reconhecimento de Expressões Faciais ser um teste análogo a um gradiente de generalização (ou teste de generalização) para estímulos envolvidos em interações sociais.

Um aspecto importante da Tarefa de Reconhecimento de Expressões Faciais é que as respostas dos sujeitos são fornecidas após uma comparação entre as faces apresentadas e os nomes que servem para classificar esses estímulos. Tendo em vista essa característica da tarefa analisada, é possível levantar a possibilidade de que alguns processos comportamentais verbais estejam envolvidos nela.

\section{Tarefa de Reconhecimento de Expressões Faciais e linguagem: procedimento de nomeação?}

No tópico anterior, foi apontado que na Tarefa de Reconhecimento de Expressões Faciais um sujeito deve classificar os estímulos apresentados com base em nomes de emoções disponíveis no teclado do computador. A partir desse aspecto, levantou-se a possibilidade de que a tarefa analisada envolve um processo comportamental verbal: a nomeação. Segundo Catania (1999), a nomeação poderia ser descrita como:

geralmente são determinados pela apresentação sucessiva de estímulos, mas em ordem irregular, em extinção" (p. 406). 
classe de ordem superior que envolve classes de estímulos arbitrários (coisas ou eventos com nomes particulares) e topografias verbais arbitrárias correspondentes (as palavras que servem como seus nomes) em uma relação bidirecional. Os pré-requisitos para a nomeação incluem pelo menos três componentes: (i) o comportamento do ouvinte, de olhar para as coisas e apontar com base no que é dito; (ii) o comportamento ecóico, repetir nomes quando eles são falados; e (iii) o tatear, isto é, dizer os nomes dados a objetos. A nomeação é gerada a partir de interações cotidianas entre as crianças e os que tomam conta delas. Uma vez que esteja disponível no repertório como uma classe de ordem superior, a nomeação favorece as expansões de vocabulário, no sentido de que a introdução de novas palavras em relações funcionais particulares (tais como as de tato) envolve essas palavras em uma ampla variedade de outras funções emergentes. (pp. 411-412)

Quando falamos de nomeação, estamos falando de um tipo especial de comportamento: o comportamento verbal (Catania, 1999). A análise do comportamento verbal é feita por meio da observação dos aspectos funcionais da linguagem ${ }^{5}$ humana. $\mathrm{O}$ foco está nas condições sob as quais as classes de respostas verbais são modeladas e mantidas, além das consequências que isso tem no comportamento do sujeito que o emitiu e em outros sujeitos que fazem parte de uma comunidade verbal ${ }^{6}$ (Catania, 1999; Skinner, 1957).

Segundo Catania (1999), respostas verbais são distinguidas com base nas ocasiões em que ocorrem e pelas consequências que produzem. Respostas verbais podem ser ocasionadas por estímulos verbais ou não verbais, assim como podem ter consequências verbais e não verbais (Catania, 1999).

5 É bom lembrar que verbal é um termo amplo que aplica-se à linguagem em qualquer modalidade (e.g., falada e escrita). Esse termo deve ser distinguido do termo vocal, que se restringe à linguagem falada (Catania, 1999).

6 É importante colocar que, para Catania (1999), uma análise funcional pode ser complementada por uma análise estrutural da linguagem, embora na literatura esses dois tipos de abordagem tenham sido contrapostos e/ou colocados como incompatíveis.
Chamamos de tato respostas verbais ocasionadas por estímulos discriminativos não verbais, quando estes estão presentes ou quando a resposta ocorre logo após sua apresentação. Esse não é um nome de um processo de controle de estímulos novo ou diferente: é apenas um nome para o controle de estímulo envolvido no comportamento verbal (Catania, 1999). Embora não sejam exatamente a mesma coisa, o tato pode ser um componente da nomeação (Catania, 1999)7.

$\mathrm{Na}$ Tarefa de Reconhecimento de Expressões Faciais, a nomeação é justamente ocasionada pela observação de um estímulo discriminativo não verbal. Desta maneira, poderíamos especificar que a nomeação do sujeito nessa tarefa envolve justamente o componente tato, na medida em que um estímulo não verbal (i.e., as faces) ocasiona uma resposta verbal que o descreve (i.e., nome da emoção mostrada na face), sendo que esta resposta é fornecida logo em seguida à apresentação do estímulo.

A hipótese de que o componente da nomeação avaliado na Tarefa de Reconhecimento de Expressões Faciais é o tato poderia ser fortalecida com outro argumento, este relacionado ao procedimento adotado nessa tarefa. As faces são apresentadas por um período muito curto de tempo (500ms) e o sujeito é solicitado a responder o mais rapidamente possível. Esses dois aspectos impedem (ou ao menos dificultam bastante) que as respostas sejam dadas baseadas em um julgamento mais demorado dos estímulos, isto é, baseado em outras respostas emitidas pelo sujeito, (em termos leigos, diríamos que o sujeito não tem tempo para "pensar sobre os estímulos"). Assim, é muito provável que as respostas sejam fornecidas com base nos estímulos mostrados e não a partir de outras respostas verbais que poderiam ser ocasionadas caso houvesse tempo para examiná-los.

Um detalhe importante é que aquilo que é tateado depende das condições vigentes no momento em que este comportamento ocorre. Por exemplo, podemos dizer o aroma ou a cor de uma fruta, o

7 Diferentemente da nomeação, o tato pode ocorrer somente na presença do estímulo tateado ou logo em seguida à sua apresentação; por outro lado, podemos nomear um objeto que está ausente. Assim, embora o tato possa ser um componente daquilo que chamamos de nomeação, a distinção ainda é mantida (Catania, 1999). 
que será dito dependerá da ocasião (por exemplo, se nos foi perguntado qual é a cor ou qual é o aroma) (Catania, 1999). A ocasião ou estímulo que determina o que será estímulo discriminativo é denominado estímulo condicional (Catania, 1999). No caso da Tarefa de Reconhecimento de Expressões Faciais, as instruções fornecidas poderiam ser consideradas como estímulo condicional que ocasiona o tatear de alguma propriedade específica do estímulo face (no caso, qual é a emoção que está sendo retratada).

\section{Propostas de pesquisa e possível aplicabilidade da Tarefa de Reconhecimento de Expressões Faciais em pesquisas com enfoque analítico- comportamental}

Em primeiro lugar, poderíamos nos perguntar se o desempenho de um sujeito na Tarefa de Reconhecimento de Expressões Faciais poderia mudar em função da exposição a procedimentos comportamentais específicos. Por exemplo, seria interessante observar se o reconhecimento de expressões faciais muda em função da exposição a protocolos experimentais como, por exemplo, aquele usado em pesquisas sobre desamparo aprendido em humanos (Samelo, 2008). Outro uso possível seria em pesquisas aplicadas. Por exemplo, seria possível usar essa tarefa para avaliar os efeitos de um procedimento de discriminação para indivíduos com dificuldades de interação social (e.g., sujeitos com desenvolvimento atípico ou fobia social) ou algum procedimento de reforçamento social? Ficariam os sujeitos mais sensíveis a determinadas expressões faciais de emoção após certos treinos de discriminação ou reforçamento social? Em caso afirmativo, seria esta uma tarefa útil para clínicos que atuam nessa área? Teria ela um valor preditivo do quanto o treino funcionará na vida do sujeito (um valor "ecológico", por assim dizer)?

Uma outra possibilidade seria a de utilizar essa tarefa em populações específicas para avaliar o controle discriminativo de estímulos envolvidos em interações sociais. Poderíamos nos perguntar se e como o desempenho de um sujeito nessa tarefa varia em função de diferentes histórias de interação social ao longo da vida. Embora seja muito difícil classifi- car ou categorizar histórias de interação social entre humanos de maneira rigorosa ou controlada, ainda assim é possível identificar alguns padrões mais ou menos estáveis para algumas populações clínicas. Por exemplo, poderíamos nos perguntar se um sujeito exposto sistematicamente a situações de violência relacionadas à interação social (e.g., abuso na infância ou adolescência e permanência em instituições prisionais) teria um desempenho diferente nessa tarefa em comparação a um sujeito sem esse tipo de história. Nesses casos, seriam esses sujeitos mais "sensíveis" a expressões faciais de emoções negativas (e.g., medo, raiva e nojo)?

Também poderíamos nos perguntar sobre o efeito de eventos "agudos" no reconhecimento de expressões faciais como, por exemplo, traumas. Mais especificamente, seria interessante utilizar esse procedimento com sujeitos expostos a eventos traumáticos (e.g., violência urbana e guerras) e verificar se há diferenças em relação a sujeitos que não foram expostos a tais fatores, sejam eles indivíduos com ou sem Transtorno do Estresse PósTraumático.

A análise dos processos comportamentais básicos envolvidos na Tarefa de Reconhecimento de Expressões Faciais permite levantar diversas questões e perguntas de pesquisa. Por outro lado, essa análise também pode permitir o exame de uma questão que tem sido discutida em análise do comportamento, aquela referente a como as drogas antidepressivas funcionam. As pesquisas utilizadas para a descrição da tarefa buscam justamente essa compreensão. Assim, o entendimento de que há processos comportamentais básicos envolvidos na Tarefa de Reconhecimento de Expressões Faciais pode auxiliar na compreensão da função comportamental de medicações psiquiátricas? Se a Tarefa de Reconhecimento de Expressões Faciais é um teste de controle de estímulos e é justamente esse controle de estímulos que muda quando da alteração do sistema serotoninérgico por antidepressivos ou outras intervenções, o que isso nos diz sobre a função comportamental dos antidepressivos? Poderíamos falar da droga como operação estabelecedora?

Até aqui pensamos em perguntas de pesquisas baseadas na Tarefa de Reconhecimento de Expressões Faciais da maneira como a usou Harmer, Bhagwagar, et al. (2003), Harmer, Hill, et 
al. (2003), Harmer, Rogers, et al. (2003) e Harmer et al. (2004). Contudo, nada impede que ela sofra modificações para o seu uso em pesquisas com enfoque analítico-comportamental. Por exemplo, uma possibilidade de modificação seria utilizar gradações de expressões de emoções que fossem de uma emoção até outra emoção e não de uma emoção até uma face neutra, como o fazem Young et al. (1997).

Em suma, parece ser viável o uso da Tarefa de Reconhecimento de Expressões Faciais para estudos sob enfoque analítico-comportamento. Algumas poucas foram delineadas acima, porém certamente há mais possibilidades.

\section{Considerações finais}

Este trabalho procurou se constituir como um conjunto de sugestões quanto ao entendimento de um paradigma neuropsicológico sob a ótica analítico-comportamental e um questionamento quanto à sua aplicabilidade em pesquisas nesta abordagem. Analisamos a chamada Tarefa de Reconhecimento de Expressões Faciais (Harmer, Rogers, et al. 2003), paradigma neuropsicológico amplamente usado na literatura em neurociências para medir aquilo que tem sido denominado processamento de emoções. A partir das categorias de análise escolhidas (Catania, 1999), levantamos a possibilidade de que esta seja uma tarefa que examina o controle de estímulos envolvidos em interações sociais. Especificamente, a tarefa foi pensada como um teste análogo a um gradiente de generalização (ou teste de generalização) para estímulos envolvidos em interações sociais, no qual o sujeito deve nomear estímulos para os quais foi treinado a responder diferencialmente ao longo da vida.

As análises realizadas parecem plausíveis e permitiram o levantamento de alguns problemas de pesquisa relevantes. No entanto, é importante colocar que elas não excluem de modo algum outras possibilidades de interpretação. Em primeiro lugar, as faces foram consideradas aqui dentro de um contexto de controle de estímulos, tendo em vista que os sujeitos devem classificá-las. No entanto, elas também podem ser consideradas a partir de outras funções operantes como, por exemplo, estímulos aversivos ou apetitivos. Essa análise poderia ser feita a partir de outros testes, nos quais há a possibilidade de observar respostas de fuga ou aproximação frente aos estímulos. De fato, há estudos que se utilizam de paradigmas nos quais o sujeito pode emitir respostas de fuga/aproximação, seja usando um joystick, seja desviando/dirigindo o olhar para as faces (Marsh, Ambady \& Kleck, 2005; Mogg \& Bradley, 2002). Uma análise desses procedimentos poderia se mostrar útil para o pesquisador que busca entender outras funções operantes das expressões faciais de emoção. De fato, essa análise permitiria discutir com mais profundidade a denominação das faces de medo como estímulos aversivos (Ekman, Sorenson \& Friesen, 1969).

Outro aspecto a ser destacado é que aqui se assumiu o papel de discutir a função operante das faces no procedimento analisado. Contudo, a apresentação de faces pode também ter funções respondentes. Existem alguns estudos que se aproximam dessa ideia. Por exemplo, há pesquisas que investigam alterações em medidas fisiológicas quando do reconhecimento de expressões faciais de emoções específicas (Ekman, Levenson \& Friesen, 1983; Levenson, Ekman \& Friesen, 1990). Esse também poderia ser um tema de potencial interesse ao analista do comportamento.

Como colocado por Strapasson et al. (2007), a interpretação de termos de outras tradições da psicologia constitui um exercício conceitual/teórico que por si só é insuficiente para o crescimento e sobrevivência da $\mathrm{AC}$ enquanto prática cultural. Para se tornar uma contribuição de fato, essas interpretações devem ser seguidas de pesquisas instigadas pelas traduções comportamentais (Strapasson et al., 2007). Embora este trabalho não tenha analisado um conceito ou termo, mas sim um procedimento, o princípio também procede aqui. As análises conduzidas ao longo deste trabalho permitiram o levantamento de alguns problemas de pesquisa de potencial interesse ao analista do comportamento e apontaram alguns caminhos possíveis de investigação. Todas essas hipóteses deverão ser confirmadas por meio de estudos empíricos no futuro. 


\section{Referências}

Andrew, R. J. (1963). Evolution of Facial Expression. Science, 142, 1034-1041.

Attenburrow, M. J., Williams, C., Odontiadis, J., Reed, A., Powell, J., Cowen, P. J., \& Harmer, C. J. (2003). Acute administration of nutritionally sourced tryptophan increases fear recognition. Psychopharmacology, 169, 104-107.

Browning, M., Reid, C., Cowen, P. J., Goodwin, G. M., \& Harmer, C. J. (2007). A single dose of citalopram increases fear recognition in healthy subjects. Journal of Psychopharmacology, 21, 684-690.

Catania, A. C. (1999). Aprendizagem: Comportamento, linguagem e cognição. 4 ed. Porto Alegre: Editora Artmed.

Corchs, F. (2010). É possível ser um psiquiatra behaviorista radical? Primeiras reflexões. Revista Perspectivas, 1(1), 55-66.

Darwin, C. (1872). The expression of the emotions in man and animals. Definitive Edition. New York: Oxford University Press.

Del-Ben, C. M. (2008). Processamento de expressões faciais de emoções básicas: Modulação farmacológica, diferenças entre gêneros e fases do ciclo menstrual. Tese de Livre Docência, Faculdade de Medicina de Ribeirão Preto, Ribeirão Preto.

Del-Ben, C. M., Ferreira, C. A. Q., Alves-Neto, W. C., \& Graeff, F. G. (2008). Serotoninergic modulation of face-emotion recognition. Brazilian Journal of Medical and Biological Research, 41, 263-269.

de Rose, J. C. (1999). O que é um skinneriano? Uma reflexão sobre mestres, discípulos e influência intelectual. Revista Brasileira de Terapia Comportamental e Cognitiva, 1, 67-74.

Dismoor, J. A. (1983). Observing response and conditioned reinforcement. The Behavioral and the Brain Sciences, 6, 693-704.

Ekman, P. (1972). Universals and cultural differences in facial expressions of emotion. In J. cole (ed.), Nebraska Symposium on Motivation, 1971. Lincoln, NE: University of Nebraska Press, pp. 207-283.

Ekman, P. (1973). Cross-cultural studies of facial expression. In P. Ekman (ed.), Darwin and facial expression: A century of research in review.
New York: Academic Press, pp. 169-222.

Ekman, P. (1979). About brows: Emotional and conversational signals. In M. von Cranach, K., Foppa, W. Lepenies, \& D Ploog (eds), Human Ethology. Cambridge: Cambridge University Press, pp. 169-248.

Ekman, P. (1992). Facial Expression of emotion: New findings, new questions. Psychological Science, 3, 34-48.

Ekman, P. (1999). Facial Expressions. In Chap 16 T. Dalgleish \& M. Power, Handbook of cognition and emotion. New York: John Wiley \& Sons Ltd.

Ekman, P., \& Friesen, W. V. (1971). Constants across cultures in the face and emotion. Journal of Personality and Social Psychology, 17, 124129.

Ekman, P., \& Friesen, W. V. (1976). Pictures of Facial Affect. Palo Alto, CA: Consulting Psychologists' Press.

Ekman, P., Friesen, W. V., \& Tomkins, S. S. (1971). Facial affect scoring technique: A first validity study. Semiotica, 2, 37-58.

Ekman, P., Levenson, R. W., \& Friesen, W. V. (1983). Autonomic nervous system activity distinguishes between emotions. Science, 221, 1208-1210.

Ekman, P., Sorenson, E. R., \& Friesen, W. V. (1969). Pan-cultural elements in facial displays of emotion. Science, 164(3875), 86-88.

Ekman, P., Friesen, W. V., O'Suliivan, M., Chan, A., Diacoyanni-Tarlatzis, I., Heider, K., \& Tzavaras, A. (1987). Universals and cultural differences in the judgements of facial expressions of emotion. Journal of Personality and Social Psychology, 53, 712-717.

Ferster, C. B., Culbertson, S., \& Boren, M. C. P. (1968/1977). Princípios do comportamento. (Trads M. I. Rocha e Silva, M. A. C. Rodrigues e M. B. L. Pardo) São Paulo: Editora Hucitec/ Edusp.

Fusar-Poli, P., Placentino, A., Carletti, F., Landi, P., Allen, P., Surguladze, S., Benedetti, F., Abbamonte, M., Gasparotti, R., Barale, F., Perez, J., McGuire, P., \& Politi, P. (2009). Fuctional atlas of emotional faces processing: A voxel-based meta-analysis of 105 functional magnetic resonance imaging studies. Journal of Psychiatry and Neuroscience, 34, 418-432. 
Guapo, V. G., Graeff, F. G., Zani, A. C. T., Labate, C. M., dos Reis, R. M., \& Del-Ben, C. M. (2009). Effects of sex hormonal levels and phases of the menstrual cycle in the processing of emotional faces. Psychoneuroendocrinology, 34, 1087-1094.

Green, L. (2006). Stasis and change. Journal of the Experimental Analysis of Behavior, 85, 1-2.

Harmer, C. J. (2008). Serotonin and Emotional Processing: does it help explain antidepressant drug action? Neuropharmacology, 55, 1023 1028.

Harmer, C. J., Rogers, R. D., Tunbridge, E., Cowen, P. J., \& Goodwin, G. M. (2003). Tryptophan depletion decreases the recognition of fear in female volunteers. Psychopharmachology. 167(4), 411-417.

Harmer, C. J., Bhagwagar, Z., Perret, D. I., Vollm, B. A., Cowen, P. J., \& Goodwin G. M. (2003). Acute SSRI administration affects the processing of social cues in healthy volunteers. Neuropsychopharmacology, 28, 148-152.

Harmer, C. J., Hill, S. A., Taylor, M. J., Cowen, P. J., \& Goodwin, G. M. (2003). Towards a neuropsychological theory of antidepressant drug action: potentiation of norepinephrine activity increases positive emotional bias. American Journal of Psychiatry, 160, 990-992.

Harmer, C. J., Shelley N. C., Cowen, P. J., \& Goodwin, G. M. (2004). Increased positive versus negative affective perception and memory in healthy volunteers following selective serotonin and norepinephrine reuptake inhibition. American Journal of Psychiatry, 161, 1256-1263.

Hayward, G., Goodwin, G. M., Cowen, P. J., \& Harmer, C. J. (2005). Low-dose tryptophan depletion in recovered depressed patients induces changes in cognitive processing without depressive symptoms. Biological Psychiatry, 57, 517-524.

Horstmann, G. (2003). What do facial expressions convey: feeling states, behavioral intentions, or action requests? Emotion, 3, 150-166.

Hunziker, M. H. L. (2011). Afinal, o que é controle aversivo? Acta Comportamentalia: Revista Latina de Análisis de Comportamiento, 19, 9-19.

Lattal, A. K. (2005). Ciência, Tecnologia e Análise do Comportamento. In J. AbreuRodrigues \& M. R. Ribeiro (Orgs.), Análise do
Comportamento: Pesquisa, Teoria e Aplicação (pp. 15-26). São Paulo, SP: ArtMed.

Lattal, A. K. (2008). JEAB at 50: Coevolution of research and technology. Journal of the Experimental Analysis of Behavior, 89, 129-135.

Levenson, R. W., Ekman, P., \& Friesen, W.V. (1990). Voluntary facial action generates emotion-specific autonomic nervous system activity. Psychophysiology, 27, 363-384.

Lezak, M., Howieson, D. B., \& Loring, D. W. (2004). Neuropsychological assessment. fourth edition. New York: Oxford University Press.

Marr, M. J. (1984). Conceptual approaches and issues. Journal of the Experimental Analysis of Behavior, 42, 353-362.

Marsh, A. A., Ambady, N., \& Kleck, R. E. (2005). The effects of fear and anger facial expressions on approach- and avoidance-related behaviors. Emotion, 5(1), 119-124.

Mogg, K., \& Bradley, B. P. (2002). Selective orienting of attention to masked threat faces in social anxiety. Behavior Research and Therapy, 40, 1403-1414.

Russel, J. A. (1994). Is there universal recognition of emotion from facial expression? A review of cross-cultural studies. Psychological Bulletin, $115,102-141$.

Samelo, M. J. (2008). Investigação sobre o desamparo aprendido em humanos. Dissertação de Mestrado, Instituto de Psicologia, Universidade de São Paulo, São Paulo.

Skinner, B. F. (1953). Science and Human Behavior. New Your, NY: The Free Press.

Skinner, B. F. (1957). Verbal Behavior. New York: Appleton-Century-Crofts.

Skinner, B. F. (1981/2007). Seleção por conseqüências. Revista Brasileira de Terapia Comportamental e Cognitiva, 9(1), 129-137.

Strapasson, B. A., Carrara, K., \& Lopes Júnior, J. (2007). Consequências da interpretação funcional de termos psicológicos. Revista Brasileira de Terapia Comportamental e Cognitiva, 9(2), 73-85.

Surguladze, S. A., Senior, C., Young A. W., Brébion, G., Travis, M. J., \& Phillips, M. L. (2004). Recognition accuracy and response bias to happy and sad facial expressions in patients with major depression. Neuropsychology, 18(2), 212-218. 
Tomanari, G. (2009). Resposta de observação: Uma reavaliação. Acta Comportamentalia, 17, 259277.

Wray, A. M., Freund, R. A., \& Dougher, M. J. (2009). A behavior-analytic account of cognitive bias in clinical populations. Behavior Analyst, 32, 2949.

Young, A. W., Rowland, D., Calder, A. J., Etcoff, N. L., Seth, A., \& Perrett, D. I. (1997). Facial expression megamix: Tests of dimensional and category accounts of emotion recognition. Cognition, 63, 271-313.

Informações do Artigo

Histórico do artigo:

Submetido em: 20/04/2013

Primeira decisão editorial: 16/06/2013

Segunda decisão editorial: 24/06/2013

Aceito em: 02/07/2013 